\title{
Gestión Didáctica en Clases y su Relación con las Decisiones del Profesor: el caso del Teorema de Pitágoras en séptimo grado*
}

\section{Classroom Didactic Management and its Relation with Teachers' Decisions: the case of the Pythagorean Theorem in seventh-grade}

\author{
Raimundo Olfos $^{* *}$ \\ Ismenia Guzmán ${ }^{* * *}$ \\ Soledad Estrella ${ }^{* * * *}$
}

\begin{abstract}
Resumen
Este artículo presenta el análisis de la gestión didáctica de dos profesoras de matemáticas en Chile, a partir de la observación directa de sus clases de geometría del teorema de Pitágoras, en el grado 7 y alumnos de 12 a 13 años. El objetivo del estudio consiste en detectar, en la gestión de la clase, los índices de las decisiones didácticas que toman las docentes sobre el tema, y verificar si sus gestiones siguen algún modelo didáctico de la acción pedagógica. Este estudio se centra en el análisis de episodios seleccionados de las clases, el cual se inscribe en una perspectiva constructivista del aprendizaje y de la enseñanza de la matemática, basado en ideas claves de la teoría de situaciones didácticas de Brousseau. La gestión de los profesores conduce a un modelo de transmisión de conocimientos que dificultan el desarrollo de las matemáticas y el protagonismo del estudiante.
\end{abstract}

Palabras-clave: Teoría de Situaciones Didácticas. Estudio de Casos. Teorema de Pitágoras. Gestión Didáctica.

\begin{abstract}
This paper reports the analysis of the didactical management of two mathematics teachers in Chile, from direct observation of their geometry classes about the Pythagorean Theorem, in grade 7 and students 12 to 13 years old. The aim of the study is to detect in classroom management, indicators of teachers'didactical decisions about the topic and see if their efforts follow a didactic model of pedagogical action. This study focuses on the analysis of selected episodes of the lessons, which is part of a constructivist approach to learning and teaching of mathematics, based on key ideas of the theory of didactic situations of Brousseau. The management of both teachers leads to a transmission model of knowledge that hampers the development of mathematics and student protagonism.
\end{abstract}

Keywords: Theory of Didactical Situations. Case Studies. Pythagorean Theorem. Didactic Management.

\footnotetext{
* Se agradece a CONICYT Chile el financiamiento de los Proyectos FONIDE N¹0980, PIA CONICYT CIE-05 del Centro de Investigación Avanzada en Educación y FONDECYT No 1111009.

** Doctor por University of Wales. Profesor Adjunto del Instituto de Matemática de la Pontificia Universidad Católica de Valparaíso, Valparaíso, Chile. Dirección Postal: Blanco Viel 596, Cerro Barón, Valparaíso, Chile. E-mail: raimundo.olfos@ucv.cl.

**** Doctora por Universidad Louis Pasteur de Estrasburgo. Profesora Universidad de Los Lagos, Chile. Dirección Postal: Avda. República 517, Santiago, Chile.E-mail: iguzmanr@ucv.cl, ismenia.guzman@ulagos.cl.

**** Doctora por Pontificia Universidad Católica de Valparaíso. Profesora Asociada del Instituto de Matemática de la Pontificia Universidad Católica de Valparaíso, Valparaíso, Chile. Dirección Postal: Blanco Viel 596, Cerro Barón, Valparaíso, Chile.E-mail: soledad.estrella@gmail.com.
} 


\section{Problemática}

Esta investigación surge del proyecto $\mathrm{FONIDE}^{1}$, que indagó la asociación entre el conocimiento del profesor, tanto de naturaleza disciplinaria como didáctica, su gestión en el aula y los saberes que dominan sus alumnos. La investigación dio evidencias de que el conocimiento disciplinario tiene una asociación positiva pero muy baja con respecto a los aprendizajes de los alumnos. El conocimiento didáctico del profesor mostró mayor asociación, pero también baja, con los aprendizajes de sus alumnos.

En consecuencia, en la presente investigación, nos preguntamos de qué depende la gestión del conocimiento en el aula por parte del profesor, cuáles son las variables que maneja y que influyen en esta gestión. Pretendemos dilucidar esta problemática, identificando algunas de esas variables mediante la observación de dos profesoras en clases de geometría sobre el Teorema de Pitágoras.

En Chile, el Programa de Matemáticas del grado 7 del Ministerio de Educación (MINEDUC, 2000) presenta el tratamiento del Teorema de Pitágoras en la siguiente forma Investigación sobre aplicaciones prácticas del Teorema de Pitágoras - y, respecto al aprendizaje esperado, señala: Utilizan de manera pertinente el Teorema de Pitágoras para la resolución de problemas cotidianos, del ámbito de otras disciplinas y de oficios.

En las Orientaciones Didácticas de dicho programa se propone conectar las potencias con actividades relativas a este Teorema, esencialmente se trata que los alumnos comprendan las relaciones de los lados de un triángulo rectángulo cualquiera y las apliquen a situaciones de cálculo de distancias no susceptibles de ser medidas directamente. Además, sugiere proponer a los alumnos realizar indagaciones sobre el uso del Teorema de Pitágoras en el trabajo práctico de los carpinteros y albañiles, quienes lo utilizan con propiedad para construir ángulos rectos.

Respecto a las actividades sobre el Teorema de Pitágoras, el programa expresa "Desarrollan y analizan procedimientos numéricos para verificar que la suma de las áreas de los cuadrados construidos a partir de los catetos es equivalente a la del cuadrado construido sobre la hipotenusa de un triángulo rectángulo cualquiera" (MINEDUC, 2000, p. 144); "Utilizan el Teorema de Pitágoras para la resolución de problemas de cálculo de distancias" (MINEDUC, 2000, p. 145); e "Investigan sobre aplicaciones del Teorema de Pitágoras en

\footnotetext{
${ }^{1}$ FONIDE, Fondo de Investigación y Desarrollo en Educación, №f4-10980, "Conocimiento pedagógico del contenido y su incidencia en la enseñanza de la matemática, nivel de educación básica" (OLFOS; GUZMÁN; GALBIATI, 2010).
} 
situaciones cotidianas como, por ejemplo, la verificación de ángulos rectos. Establecen conclusiones respecto de los números pitagóricos” (MINEDUC, 2000, p. 148).

\title{
2 Marco teórico
}

De la teoría de situaciones de Guy Brousseau hemos considerado los conceptos fundamentales en relación a la gestión de clases $^{2}$ : el contrato didáctico, los procesos de devolución y de institucionalización, y la paradoja pedagógica conocida como efecto Topaze, estos como índices de las decisiones didácticas de las docentes, entre otros.

Respecto al contrato didáctico, Brousseau (1998, p. 60) afirma:

\begin{abstract}
El contrato didáctico es la regla del juego y la estrategia de la situación didáctica (situación problema o situación de enseñanza prevista); es el medio que tiene el maestro de ponerla en escena. Pero la evolución de la Situación modifica el contrato lo cual permite a su vez la obtención de situaciones nuevas (nuevas tareas, nuevas preguntas). De esta manera, el conocimiento es lo que se expresa a través de las reglas de la situación y de las estrategias. La evolución de esas estrategias requiere producciones de conocimientos que a su vez permiten la concepción de nuevas situaciones adidácticas (situaciones problemas o verdaderos problemas escolares). El contrato didáctico depende estrechamente de los conocimientos en juego y no es un contrato pedagógico general.
\end{abstract}

Según Brousseau (1986), la enseñanza exige a los profesores elegir verdaderas situaciones problemas, las que deben presentar características tales que permitan a los alumnos de mutuo propio: reaccionar, buscar caminos, comunicar, probar y controlar. A estas situaciones problemas, él las denomina situaciones adidácticas, las cuales están asociadas a un conocimiento (nuevo para los alumnos), y su gestión en clases está asociada con las actividades o tareas que los alumnos (de mutuo propio) deben realizar. Todo regido por el contrato didáctico que el profesor va manejando en el desarrollo de la situación, poniendo en juego el proceso de devolución.

Respecto a la devolución, Brousseau (1998, p. 60) afirma,

El maestro busca la manera de hacer la devolución al alumno de una situación adidáctica de modo que le provoque la interacción, la más independiente y la más fecunda posible. Para esto, él comunica o no, según el caso, informaciones, preguntas, métodos de aprendizaje, heurísticas, etc. El maestro entonces está implicado en un juego entre el sistema de interacciones del alumno con el problema que él le ha planteado [...].

En otras palabras, en el proceso devolución pone en juego dos tipos de interacciones, las del alumno con el problema y las del alumno con el profesor respecto al problema. El profesor, a través de preguntas en relación con el conocimiento previsto, lleva el proceso de

\footnotetext{
${ }^{2}$ El lector puede remitirse al texto original en francés, en el Anexo.
} 
enseñanza de modo de favorecer los aprendizajes. De esta manera, el proceso de devolución plantea una diferencia absoluta entre la gestión de una enseñanza conducida por el profesor y la gestión de una enseñanza centrada en la actividad del alumno juiciosamente planificada.

Otra de las nociones de la teoría a la que hemos recurrido para nuestro análisis son las paradojas pedagógicas; la más común es el efecto Topaze, nombre que Brousseau (1998, p. 52) ha tomado de la novela de Marcel Pagnol. La escena que lo inspira es la siguiente:

Topaze hace un dictado a un alumno [de malas notas] y no pudiendo aceptar los errores garrafales del alumno y no pudiendo tampoco indicar directamente la ortografía pedida, él sugiere la respuesta, disimulándola con códigos didácticos cada vez más transparentes $[\ldots]$.

El efecto Topaze ilustra la relación entre las respuestas solicitadas a los alumnos y la acción docente. En esencia, la respuesta que se espera debe ser consecuencia de un aprendizaje, pero lo que hace Topaze es contrario a una verdadera acción docente. Una respuesta dada por un alumno y que ha sido sugerida por el docente por signos o cuestiones nemotécnicas, no da cuenta de un aprendizaje.

Más adelante el autor agrega:

La respuesta que debe dar el alumno está previamente determinada, el maestro elige las preguntas para las cuales esa respuesta conviene. Los conocimientos necesarios para producir esas respuestas pueden evidentemente cambiar también su significación. Haciendo preguntas cada vez más fáciles, el maestro trata de obtener la significación máxima para el máximo de alumnos. Cuando los conocimientos previstos desaparecen completamente, se trata del 'Efecto Topaze' (BROUSSEAU, 1998, p. 52).

En relación con la Institucionalización, Brousseau (1998, p. 311) afirma:

El profesor tiene que constatar lo que los alumnos tenían que hacer (o rehacer) o no, lo que han aprendido o lo que tenían que aprender. Esta actividad es insoslayable: no se puede reducir la enseñanza a la organización de aprendizajes. La toma de conciencia del objeto de conocimiento "oficial", tanto por parte de los alumnos como del profesor, es un fenómeno social muy importante y una fase esencial del proceso didáctico: este doble reconocimiento es el objeto de la institucionalización. También es rol del profesor institucionalizar.

En el modelo de enseñanza tradicional, la institucionalización está contenida en la exposición del profesor, pero, en general, el alumno no participa en ella. En cambio en los modelos inspirados en la construcción de los conocimientos, el proceso de enseñanza está centrado en la actividad de los alumnos, por lo tanto su participación es esencial, pero muchas veces el profesor pierde el rumbo en la gestión de la clase y no oficializa los conocimientos puestos en juego ni los emergentes. 


\section{Metodología}

Hemos realizado un estudio de casos, a través de una observación directa a dos profesoras sobre el Teorema de Pitágoras en clases en el grado 7. Una de las profesoras tenía formación inicial en Educación General Básica, con 31 años de experiencia en el nivel, y en los últimos cuatro años ha seguido una formación continua sistemática en didáctica de la matemática. La otra docente tenía formación inicial como profesora de matemática en Educación Media, con 35 años de experiencia en el nivel, y no ha participado en cursos de perfeccionamiento en los últimos cuatro años. El curso de la docente de Educación Básica tenía 30 alumnas y el curso de la docente de Educación Media era mixto y también de 30 alumnos.

Ambas profesoras pertenecían a la muestra del Proyecto FONIDE, antes mencionado, y fueron elegidas por las características distintivas de sus formaciones y por estar tratando el teorema de Pitágoras en el mismo nivel de enseñanza. Observamos dos clases continuas sobre el tema a cada profesora, con apoyo de notas de campo y videograbaciones.

\section{Desarrollo de las observaciones}

Cada observación la organizamos en episodios, es decir, momentos de la clase que consideramos esenciales debido a la acción tanto de las docentes como de los alumnos, en relación al saber puesto en juego. Presentamos los episodios seleccionados en cada observación conjuntamente con un primer análisis de los mismos. La profesora de Educación Básica la identificamos como EB y la profesora de Educación Media como EM.

A continuación, los episodios de la docente EB.

\section{Episodio 1:}

EB saluda al curso y se disculpa por su ausencia a la clase pasada. Luego pregunta ¿Qué hicieron la clase anterior? Se dirige a dos niñas de la primera fila, ellas le muestran sus cuadernos, y dicen triángulos. EB pregunta ¿qué tipo de triángulo era? Algunas alumnas responden: rectángulo.

En la clase anterior se les había pedido construir la configuración pitagórica y verificar por superposición. EB podría haber recapitulado y averiguado cómo las niñas realizaron las actividades de construcción (triángulo rectángulo y cuadrados) y de superposición. 
Consideramos que este hecho necesitaba un tratamiento explícito, dado que la superposición de los cuadrados no es directa y uno de ellos requiere transformaciones.

Episodio 2:

EB: dirigiéndose al curso pregunta ¿Conocen un triángulo acutángulo?

Als.: Algunas responden, Si.

EB: Ahora, van a construir un triángulo acutángulo equilátero. Les voy a dar las medidas de los lados. Si es un triángulo equilátero acutángulo ¿Cuál será la medida de sus lados?

Als.: Todas iguales

EB: El triángulo será equilátero, de medida $6 \mathrm{~cm}$. Una vez construido, ustedes construirán los cuadrados. Y veremos.

EB: Recorriendo las mesas mira los cuadernos y lo que hacen las niñas. Va comentando y dice a una alumna "No veo los vértices", a otra "No veo tu triángulo equilátero, debe tener la misma medida", y a otra alumna le indica "tu triángulo equilátero, debe tener la misma medida".

En este episodio notamos la falta de explicitación del objetivo de la clase. EB inicia la clase con instrucciones imprecisas y redundantes en el lenguaje (triángulo equilátero y acutángulo). Señala omisiones en los registros de las alumnas sin profundizar en un diálogo con ellas, desaprovechando un buen momento para la devolución. Aparece un efecto Topaze cuando EB dice tu triángulo equilátero, debe tener la misma medida en lugar de preguntar ¿qué características tiene un triángulo equilátero?

Episodio 3:

EB: va a la pizarra y dibuja la configuración pitagórica clásica ${ }^{3}$ con una escuadra que le facilita una alumna.

Al.: ¡Ah! Ese dibujo es lo que hicimos en la hojita [trabajo realizado en la clase anterior].

EB: da una nueva tarea. Ahora construyan la figura análoga a la que dibujé en la pizarra con el triángulo equilátero. [...]

EB: Para hacer un ángulo recto, ¿con qué trabajan?

Als.: ¡Con los catetos!

\footnotetext{
${ }^{3}$ Configuración pitagórica clásica es aquella en que se dibuja un triángulo rectángulo y los cuadrados sobre sus lados.
} 
EB: Si es un cuadrado, ¿cómo van a ser sus lados?:

Als.: ¡De igual medida!

Algunas alumnas reconocen la configuración pitagórica. Consideramos que EB deja implícitos, por ejemplo, la construcción de un cuadrado, los instrumentos a emplear, las estrategias de construcción. La interacción que sostiene con las alumnas no se relaciona con la tarea dada sino con los prerrequisitos. Ella habría podido preguntar cómo se construye un cuadrado ó con qué instrumentos ó qué estrategias conocen para construir un cuadrado. Episodio 4:

EB: [alumnas han construido los cuadrados sobre los lados del triángulo equilátero] da una nueva instrucción, Ahora recortan los cuadrados.

Al1.: ¿Cuánto miden los cuadrados?

A12.: Si ya lo dijo la profesora.

EB: Yo no lo dije, Uds. respondieron...

EB: Se acerca a otra alumna y le pregunta ¿Qué has hecho?

A13.: Nada, pero lo sé hacer.

EB: Va a la pizarra y dice "Vamos a hacer la misma actividad" y muestra la configuración pitagórica que había dibujado en la pizarra.

A lo dicho por A12, ella reacciona: Yo no lo dije, Uds. respondieron; queriendo hacer notar su identificación con un modelo pedagógico no conductista. Sin embargo, en su gestión notamos la falta de devoluciones al no interactuar con las alumnas en forma más profunda respecto a los conocimientos en juego.

La imprecisión en su instrucción, la misma actividad, deja implícito lo que ella espera, es decir la superposición.

Para las tareas de construcción las alumnas utilizaron escuadra y medidas dadas. En los casos de alumnas con dificultades en la construcción del cuadrado, EB hubiera podido reforzar los conocimientos necesarios para realizar las tareas de construcción y el uso adecuado de los instrumentos.

Episodio 5:

Al.: Dirigiéndose a la profesora ¿Para qué hacemos lo mismo? 
EB: Para ver si es lo mismo que con un triángulo rectángulo. ¡Vamos a comparar! Luego pregunta al curso, ¿Caben los dos?, [refiriéndose a la superposición de los cuadrados de los catetos sobre el cuadrado de la hipotenusa]

Als.: ¡Noo!

EB: Afirma, en el triángulo rectángulo caben los dos

A14.: Sí, pero hay que recortarlo

A15.: En el triángulo "acutángulo equilátero", no caben, uno solo cubre

EB: ¿Por qué?

A16. : Porque los cuadrados son de la misma medida

EB: Se trata de cubrir la superficie y ahora peguen los cuadrados

En este episodio, la pregunta de la alumna ¿para qué hacemos lo mismo? muestra la falta de explicitación del objetivo de la clase. Entonces ¿Cuál es el sentido para las alumnas de todas las acciones que han realizado? La docente EB dice ;Vamos a comparar! Pero le falta precisar, ¿qué se compara? Hubiera podido aprovechar la pregunta de la alumna ¿para qué hacemos lo mismo? y enfocar la clase. Por otro lado, la nueva instrucción - ahora peguen los cuadrados - deja en evidencia la ausencia de un contrato didáctico, pues las alumnas no tenían claro que hacer, ellas no fueron enfrentadas a una situación problema.

\section{Episodio 6:}

EB: Da una nueva tarea. Ahora construyan un triángulo obtusángulo de base $5 \mathrm{~cm}$ y un ángulo de 120 grados. ;Y no se demoren tanto! Va a su escritorio y espera algunos minutos. Luego pregunta: ¿Estamos construyendo?, recorre las mesas mirando lo que hacen las niñas...

A1.: ¿Cuánto es el ángulo?

A15.: jahi dice 120!

EB: ¿Puede haber otro ángulo de 120 grados? (nadie responde)

EB: Cuando digo acutángulo hablo de ángulo agudo y si digo obtusángulo hablo de ángulo obtuso.

EB: ¿Cómo son los ángulos de los cuadrados?

Als.: ¡Rectoos!

EB: Va mirando las mesas, deteniéndose en una, y pregunta ¿Es un cuadrado eso? ¿Es recto ese ángulo? ¿Qué usan para construirlo?

Als.: Escuadra 
EB: Muy bien, ¿Por qué?

\section{Al10.: Porque los lados tienen medidas diferentes}

EB: ¿Qué pasó?

Al15.: ¡No se puede cubrir!

EB: ¿Quién puede concluir?

Al15.: Solo en el triángulo rectángulo los dos caben en uno [refiriéndose a la superposición]

Observamos, nuevamente, que la docente EB no hace devoluciones, sino preguntas puntuales, empleando un lenguaje matemático insuficiente sobre acciones de las alumnas que supone sabidas, aceptando respuestas incompletas en lugar de reforzar los conceptos en juego, así la clase se ha convertido en una secuencia de instrucciones segmentadas e imprecisas.

¿Cuál era la situación central de su clase? ¿Estaba en juego la representación geométrica del Teorema de Pitágoras o su recíproco, y su verificación? La falta de institucionalización formal deja implícito el saber en juego.

Sobre esta clase pensamos que un supervisor externo - no experto en la disciplina vería un escenario en el cual se desarrolla una clase dinámica con interacciones entre los actores, y alumnas participando activamente. Sin embargo, no se percataría de la debilidad del discurso matemático de la docente y la fragilidad de su gestión que dificulta los aprendizajes de sus alumnas.

A continuación los episodios de la docente EM.

\section{Episodio 1:}

EM saluda al curso y había previsto revisar una guía de seis ejercicios, cuatro de aplicación de tríos pitagóricos y dos problemas contextualizados que había dejado de tarea.

La mayoría de los alumnos señala que no pudo realizar los problemas, a pesar de que en la clase anterior habían trabajado en los tríos pitagóricos en forma numérica con la fórmula. En consecuencia EM se ve obligada a volver atrás.

En este episodio notamos la ausencia de devolución de EM, pues, por ejemplo, podría haber preguntado ¿Qué no entiende? ¿Qué le impidió hacer el Ejercicio 1? y haber aprovechado el trabajo de los alumnos que cumplieron con la tarea propuesta, personalizando los intercambios. 


\section{Episodio 2:}

EM precisa el objetivo de la clase, “comprender las medidas mediante el teorema de Pitágoras y también que esto se da en un triángulo rectángulo; pero hay números muy especificos ¿queda claro?”

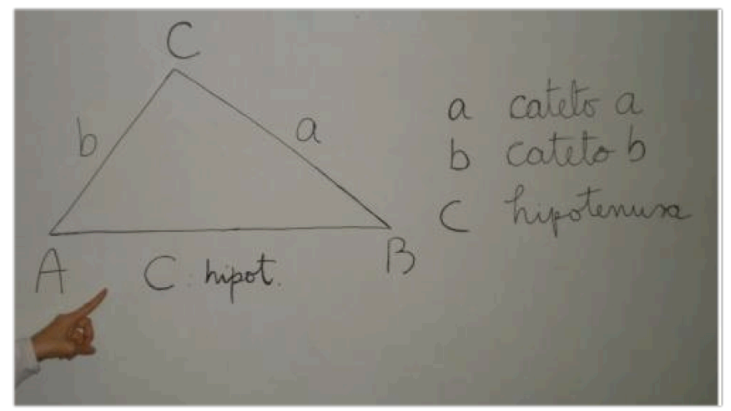

Figura 1 - Imagen de la pizarra, docente EM Fuente: los autores

Ella dibuja a mano alzada el triángulo en la pizarra que muestra la imagen, la cual se supone conocida por los alumnos.

En este episodio podemos apreciar la falta de precisión en el discurso de la docente. Nos preguntamos ¿entienden los alumnos este objetivo? ¿qué significa comprender las medidas?

EM implícitamente trata el pasaje desde la forma numérica a la interpretación geométrica de la relación pitagórica, en forma incompleta ya que no explicita que las medidas de los lados del triángulo rectángulo están representadas por los números (parámetros) a, b, c de la relación pitagórica. Notamos que EM hubiera podido distinguir los lados del triángulo (objeto geométrico) con sus medidas (objetos numéricos), por ejemplo, cuando anota las letras a y b sobre los catetos del triángulo dibujado, dado que a y b estrictamente no son los catetos, sino que representan las medidas de ellos. De este modo EM pierde la focalización de la interpretación geométrica del Teorema de Pitágoras.

\section{Episodio 3:}

EM: ¿Cómo se llaman los lados en un triángulo rectángulo?

Als.: ¡catetos!, ¡hipotenusa!

EM: Escribe en la pizarra la relación pitagórica, $a^{2}+b^{2}=c^{2}$.

¿Qué significa eso?, ¿alguien lo ha entendido? La fórmula dice que es " a” cuadrado más " $b$ " cuadrado igual a "c" cuadrado, pero qué significa para ustedes,

¿Qué significa “a” cuadrado para ti?, ¿qué es “a” para ti?

Silencio, nadie responde. 
En este episodio EM se sitúa en el ámbito geométrico, y espera que los alumnos le hayan dado sentido a las letras como medidas de los lados del triángulo, lo cual nos parece difícil, ya que EM no enunció el teorema de Pitágoras geométricamente, tomando en cuenta los cuadrados construidos sobre los lados del triángulo rectángulo, y así dar significado a los números $\mathrm{a}^{2}, \mathrm{~b}^{2}$ y $\mathrm{c}^{2}$ que representarían las áreas respectivas.

Con las preguntas de EM se está configurando un efecto Topaze, ya que la docente, con su insistencia, logrará la respuesta que espera.

\section{Episodio 4:}

EM: ¿Qué tríos pitagóricos cumplen con la fórmula?

Als.: ;tres, cuatro, cinco!

EM: ¿Qué otros números?

Als.: jocho, diez, cuatro!

EM: ... entonces sería ocho y este sería diez, ¿cuáles son los números?

¿Cuál es el trío? ¿Por cuánto multiplicas?

Al.: por dos... (Varios alumnos hablan a la vez y dicen distintos números)

EM: Si yo multiplico por dos, ¿cuánto mediría para que un número me cumpla Pitágoras?

Al.: seis

EM: repite, seis, ¿y el otro?

Als.: ocho

EM: ¿y el otro?

Al.: diez

EM: entonces ¿Cuál sería la medida ahí? (indica el triángulo de la pizarra).

Este episodio se sitúa en un ámbito numérico, en las preguntas que realiza EM demanda respuestas que dejan en evidencia su propia estrategia de búsqueda (utilizando múltiplos), en lugar de dejar a cargo de los alumnos la búsqueda de estrategias. Ellos, paso a paso, responden a las preguntas puntuales de EM. Nos surge la interrogante ¿Cuán significativas son esas respuestas puntuales para ellos? ¿Qué grado de comprensión del problema que se está resolviendo alcanzan los alumnos?

Observamos claramente que las respuestas de los alumnos son de tipo aritmético y siguen las instrucciones de la docente, desviándose del problema, por cuanto nuevamente surge un efecto Topaze. 


\section{Episodio 5:}

EM: pide a un alumno leer en su cuaderno el enunciado del Teorema de Pitágoras y la observación sobre los tríos, tratados en la clase anterior.

Al.: "En todo triángulo rectángulo el cuadrado de la hipotenusa es igual a la suma de los cuadrados de las medidas de los catetos. Observación, en geometría existe un trío de números muy específico que cumple con el teorema de Pitágoras y se obtienen valores numéricos exactos, esta clase de números se conoce como números pitagóricos".

EM: Comprueben en cuatro triángulos si realmente se cumplen con los tríos. ¿Qué trío puedo ocupar? Ahora pueden conocer perfectamente sus medidas sin hacer cálculos, simplemente sabiendo el trío. ¿En el primero se cumple el teorema de Pitágoras?

Als.: ;no...! ;siii...!

EM: ¿Sí?, ¿porque si??

Al.: Porque 15, 20, 25

$\mathrm{EM}:$ ¿qué números tienen ustedes?

Als.: 15, 20, 25

EM: ¿Qué tuvieron que hacer para ello?

Al.: ;multiplicar!

EM: ¿multiplicar o sacar un factor?

Al.: jveinticinco!

EM: ¿Se dan cuenta que sin querer estábamos repasando multiplicaciones?

Este episodio comienza con la institucionalización del conocimiento matemático en juego con la lectura del cuaderno de un alumno. EM interactúa con algunos alumnos, aunque sus preguntas están dirigidas al curso, las cuales no tienen relación directa con el enunciado leído, sino con la operatoria aritmética involucrada. Su comentario final respecto al repaso de la multiplicación está desenfocado en relación al sentido de la tarea.

Episodio 6:

EM: (tras solicitar la construcción de un triángulo rectángulo isósceles en papel lustre, se dirige a un alumno) Esto es un triángulo rectángulo isósceles, ... mira ... (EM toma regla y mide un lado)... hago lo mismo pero con este lado... y te va a dar...

Al.: Profe, no me dio aquí. 
EM: Ah, entonces... veamos ¿Cuánto sería? (la profesora marca con la escuadra las medidas) ya, aqui te tiene que dar siete coma cinco y la otra igual.

En este episodio se observa que la profesora propone trabajar con material concreto, papel lustre (plantillas cuadradas de lado $10 \mathrm{~cm}$ ), llama la atención esta actividad a esta altura de la clase y con alumnos de este nivel. En este episodio notamos la ausencia de énfasis sobre el rol del ángulo recto en el triángulo pedido. Ante las dificultades de los alumnos con la medición, EM no hace devolución, sino que ella mide con la regla en el cuaderno del alumno, y oralmente adelanta las respuestas. De este modo, la profesora le quita protagonismo al alumno lo que no favorece el aprendizaje. Además, no se dirige a todo el curso para atender las dificultades comunes.

\section{Comparación de las gestiones de las docentes}

En el Cuadro 1 comparamos las gestiones de ambas docentes, según los siguientes criterios: explicitación del objetivo de la clase, situación didáctica inicial, contrato didáctico, variables didácticas, desarrollo de la clase, devolución, discurso matemático e institucionalización.

\begin{tabular}{|l|l|l|}
\hline \multicolumn{1}{|c|}{$\begin{array}{c}\text { Criterios de } \\
\text { comparación }\end{array}$} & \multicolumn{1}{|c|}{ Docente EM } & \multicolumn{1}{|c|}{ Docente EB } \\
\hline $\begin{array}{l}\text { Explicitación del } \\
\text { objetivo de la clase }\end{array}$ & $\begin{array}{l}\text { EM ha expresado oralmente el objetivo de } \\
\text { la clase. Ella proyectaba conectar los } \\
\text { números pitagóricos con las medidas de los } \\
\text { lados de un triángulo rectángulo. }\end{array}$ & $\begin{array}{l}\text { EB no explicita el objetivo de su } \\
\text { clase, hasta el requerimiento de } \\
\text { una alumna en el episodio 5. }\end{array}$ \\
\hline $\begin{array}{l}\text { Situación didáctica } \\
\text { inicial }\end{array}$ & $\begin{array}{l}\text { EM centra su clase en el triángulo } \\
\text { rectángulo que dibuja en la pizarra } \\
\text { utilizando las notaciones convencionales: } \\
\text { vértices A, B, C y lados a, b, y c. }\end{array}$ & $\begin{array}{l}\text { EB centra su clase en la actividad } \\
\text { de construcción de un triángulo } \\
\text { equilátero, para que luego las } \\
\text { alumnas construyan cuadrados } \\
\text { sobre sus lados. }\end{array}$ \\
\hline Contrato Didáctico & $\begin{array}{l}\text { Aunque la profesora plantea el objetivo, el } \\
\text { contrato queda implícito. } \\
\text { Los alumnos no tienen claro el rol que les } \\
\text { cabe, lo que explicaría que no se hayan } \\
\text { involucrado y por ende no surgieran } \\
\text { estrategias propias. }\end{array}$ & $\begin{array}{l}\text { No hubo contrato didáctico, ni } \\
\text { planteo de objetivo de la clase. Las } \\
\text { alumnas trabajan siguiendo las } \\
\text { instrucciones de la profesora, } \\
\text { ajustándose a un contrato tácito } \\
\text { usual, en el cual el profesor dirige } \\
\text { y el alumno le sigue. }\end{array}$ \\
\hline $\begin{array}{l}\text { Variables didácticas } \\
\text { en juego }\end{array}$ & $\begin{array}{l}\text { EM tenía las medidas de los lados, como } \\
\text { variables para hacer construir diversos } \\
\text { triángulos. Sin embargo, EM no las } \\
\text { maneja convenientemente, pues ella elige } \\
\text { las medidas e indica la forma de dibujar y } \\
\text { de usar la regla; en lugar de dejar a los } \\
\text { alumnos elegir libremente las medidas de } \\
\text { los lados de los triángulos y la forma de } \\
\text { construirlos. }\end{array}$ & $\begin{array}{l}\text { EB tiene como variable los tipos } \\
\text { de triángulos. Primero propone } \\
\text { construir un triángulo equilátero } \\
\text { de 6 cm de lado después un } \\
\text { triángulo obtusángulo de ángulo } \\
\text { 120 grados. Ella hace un manejo } \\
\text { acotado de las variables, pues } \\
\text { elige los tipos de triángulos con } \\
\text { medidas particulares. }\end{array}$ \\
\hline
\end{tabular}




\begin{tabular}{|c|c|c|}
\hline Desarrollo de la clase & $\begin{array}{l}\text { EM propone una secuencia de preguntas } \\
\text { dirigidas puntuales que algunos alumnos } \\
\text { contestaban, pero sus respuestas seguían la } \\
\text { estrategia y la lógica personal de la } \\
\text { profesora. Esta dinámica de la docente } \\
\text { difícilmente podría favorecer el aprendizaje } \\
\text { previsto. En consecuencia el protagonismo } \\
\text { de los alumnos estuvo ausente, así ellos no } \\
\text { se involucraron con el contenido } \\
\text { matemático que trataba el teorema de } \\
\text { Pitágoras en su expresión algebraica y su } \\
\text { conexión con la configuración geométrica. } \\
\text { De modo que la posterior aplicación de } \\
\text { este contenido a problemas en contexto } \\
\text { resultó difícil para los alumnos. }\end{array}$ & $\begin{array}{l}\text { EB propone una secuencia de } \\
\text { instrucciones: construcción de } \\
\text { triángulos, de cuadrados sobre los } \\
\text { lados, de recorte de cuadrados, de } \\
\text { superposición, y de verificación. } \\
\text { Las alumnas trabajaban según las } \\
\text { instrucciones, pero estas } \\
\text { instrucciones se muestran } \\
\text { inconexas para muchas alumnas } \\
\text { que no se percataron de la } \\
\text { intencionalidad matemática de las } \\
\text { actividades propuestas, ya que la } \\
\text { profesora no las explícito. }\end{array}$ \\
\hline Devolución & $\begin{array}{l}\text { Prácticamente ausente y aparece el efecto } \\
\text { Topaze. Existiendo oportunidades para } \\
\text { reforzar conocimientos y favorecer } \\
\text { aprendizajes. }\end{array}$ & $\begin{array}{l}\text { Prácticamente ausente y aparece el } \\
\text { efecto Topaze. Existiendo } \\
\text { oportunidades para reforzar } \\
\text { conocimientos y favorecer } \\
\text { aprendizajes. }\end{array}$ \\
\hline $\begin{array}{l}\text { Discurso matemático } \\
\text { del profesor }\end{array}$ & $\begin{array}{l}\text { Prácticamente ausente, no explicita } \\
\text { definiciones, propiedades y el enunciado } \\
\text { del Teorema de Pitágoras es impreciso } \\
\text { (episodio 5). Además, el discurso en } \\
\text { lenguaje natural es descuidado y confuso. }\end{array}$ & $\begin{array}{l}\text { Ausente, sin enunciado del } \\
\text { Teorema de Pitágoras, y el } \\
\text { discurso en lenguaje natural es } \\
\text { limitado e impreciso. }\end{array}$ \\
\hline Institucionalización & $\begin{array}{l}\text { Faltó oficializar el saber matemático } \\
\text { escolar previsto. Esto es, relacionar la } \\
\text { expresión algebraica, los tríos de números } \\
\text { pitagóricos y la representación geométrica } \\
\text { del Teorema. }\end{array}$ & $\begin{array}{l}\text { La profesora acepta como saber } \\
\text { oficial la conclusión oral solicitada } \\
\text { a una alumna sobre las actividades } \\
\text { realizadas. }\end{array}$ \\
\hline
\end{tabular}

Cuadro 1 - Gestiones de las docentes

Fuente: los autores

Aunque ambas profesoras tienen distinta formación, sus acciones pedagógicas en clases son similares, tratan de hacer participar a sus alumnos a través de preguntas puntuales y cerradas; de este modo los alumnos han tenido pocas posibilidades de preguntar, por ello el proceso de devolución resulta insuficiente. Ellas entregan una serie de instrucciones que conducen a actividades sin proyección para los alumnos. Sus discursos se desenfocan del saber matemático y el Teorema de Pitágoras no ha cobrado la relevancia que tiene en la matemática escolar. La situación didáctica inicial no ha sido bien formulada, ella fue cambiada por una tarea poco clara y sin sentido para los alumnos. La falta de contrato didáctico y de la institucionalización del saber en juego, ha desdibujado el saber y no ha favorecido el aprendizaje. No obstante, las profesoras se atienen al programa oficial e intentan hacer trabajar a sus alumnos (as), pero ambas lo hacen a través de preguntas acotadas o instrucciones puntuales; las cuales ponen al alumno en situación de dependencia, inhibiendo su trabajo autónomo y exploratorio. 


\section{Conclusiones}

Desde un punto de vista didáctico, el análisis de la gestión de las clases de ambas docentes en la perspectiva de la Teoría de Situaciones, es decir, sus acciones intencionadas en el proceso de construcción del conocimiento, ha permitido dejar en evidencia los siguientes hechos didácticos surgidos en los distintos episodios de las clases:

- las decisiones de las docentes al inicio de las clases no establecen un contrato didáctico, puesto que no plantean claramente un problema y no fijan reglas del juego que permitan un trabajo autónomo de los alumnos(as), lo que redunda en una ausencia de estrategias personales y de interacciones. La docente EM decide partir de la forma numérica de la relación pitagórica y muestra la interpretación geométrica. Por su parte, EB decide dar instrucciones, retomando la actividad de la clase anterior;

- las docentes, al plantear preguntas cerradas a los alumnos, no dejaron espacio para la interacción ni para que ellos se involucraran con el problema, por lo tanto el proceso de devolución no tuvo lugar. En cambio, aparece reiteradamente el efecto Topaze, de modo que las respuestas que logran dar los alumnos resultan sin sentido para ellos. Este hallazgo es coincidente con lo que expresa Radovic y Preiss (2010, p. 76) sobre el discurso de los profesores, quienes señalan el "predominio de preguntas de bajo desafío cognitivo" y "baja apertura de estas preguntas";

- las docentes no institucionalizan el conocimiento que habían previsto, y por tanto no constatan, al final de sus clases, si los alumnos lo han aprendido y si este conocimiento tuvo sentido y significado matemático para ellos.

Desde el punto de vista matemático, percibimos en ambas docentes una falta de claridad del objeto de saber en juego. EM dando por sabida la expresión numérica del Teorema Pitágoras se centra en los tríos pitagóricos. Después, la ilustra con triángulos rectángulos, pero no enfatiza que los lados del triángulo (objeto geométrico) se representan por sus medidas (objetos numéricos). EM no se hace cargo de la comprensión del alumno sobre el sentido y significado del Teorema de Pitágoras.

Por su parte, EB pide construir triángulos (un equilátero y un obtusángulo) para que sobre sus lados construyan cuadrados, y enseguida recortarlos y superponerlos para verificar si se cumple la misma propiedad válida para el triángulo rectángulo. En esta clase, EB no presenta de manera explícita el objeto matemático Teorema de Pitágoras, y no lo verifica, sino que, implícitamente, ella verifica el teorema recíproco de Pitágoras, creyendo que estaba 
verificando geométricamente la relación si el triángulo es rectángulo entonces los cuadrados construidos sobre los catetos pueden superponerse sobre el cuadrado construido sobre la hipotenusa.

Del punto de vista pedagógico, ambas docentes permanecen en el nivel concreto. A pesar de que ellas se atienen al programa y respetan las actividades propuestas en él, la organización de sus clases es débil, pues sus decisiones no son explícitas y no revelan un modelo pedagógico coherente.

Del punto de vista formal, las docentes están dejando de lado las clases expositivas, EB adopta la metodología de trabajo grupal dando cabida a la interacción entre los alumnos y EM utiliza la metodología de trabajo con guías de ejercicios, favoreciendo la comunicación profesora-alumna(o). Ambas docentes aceptan preguntas, pero sus respuestas no parecieran estar impactando los conocimientos de los alumnos(as). El modelo pedagógico de transmisión de saberes aflora en la gestión de estas profesoras, bajando la tensión de incertidumbre en los alumnos, dificultando paralelamente la evolución didáctica de sus acciones pedagógicas.

Consideramos que las secuencias de instrucciones dirigidas por las docentes no gatillan el aprendizaje con sentido, a pesar de que los alumnos contestan las preguntas y cumplen con las tareas que se les encomienda. El protagonismo de los alumnos respecto al teorema de Pitágoras y respecto a su aplicación en problemas en contexto estuvo ausente en las clases observadas.

De igual manera, como muchos profesores en Chile, las profesoras observadas responden al sistema de evaluación docente actual, que propone un modelo de inicio, desarrollo y cierre. La etapa de desarrollo se caracteriza por secuencias de preguntas cerradas planteadas a los estudiantes y la supervisión del trabajo en las mesas de los alumnos. Las profesoras respetan las pautas formales en desmedro del contenido matemático que tratan. Así, una eventual evaluación de ambas clases realizada por evaluadores generales, sin especialidad en matemáticas, podrían calificarlas como muy buenas, pues responden al pauteo vigente; pero vistas por especialistas en la materia, éstas carecen de los dos componentes principales de una clase de matemática: la actividad de los alumnos y el contenido matemático explícito. 


\section{Consideraciones finales}

El análisis didáctico realizado en este estudio pone en relieve la inconsistencia entre una propuesta curricular constructivista y la gestión de las clases por parte de las docentes, quienes no logran romper con el paradigma tradicional. Por un lado, dada la comprensión limitada de las profesoras sobre el teorema de Pitágoras no les es posible brindar a sus alumnos experiencias matemáticas y fecundas, ni responder adecuadamente a las preguntas que ellos les plantean; y por otro lado, dadas las insuficiencias en su conocimiento didáctico para establecer un contrato didáctico, gestionar devoluciones proactivas y manejar en conciencia las variables didácticas, las profesoras no logran tomar decisiones que las lleven a centrar su clase en la acción del alumno y en el saber matemático. Se produce una inconsistencia entre la propuesta constructivista del currículo y los desempeños de las docentes y de sus alumnos en aula.

Se hace urgente y necesario innovar mecanismos que articulen la propuesta curricular con la gestión didáctica y los aprendizajes escolares. Ello debiera orientar la formación continua e inicial de profesores, como también los procedimientos para su promoción y selección, de modo que se concilien los paradigmas en juego y se consigan acciones que impacten la práctica cotidiana. Si la innovación compatibiliza la experiencia en aula con la reflexión sobre la implementación del currículo, se favorecerá la consolidación de un paradigma de enseñanza que dé coherencia al aprendizaje de técnicas y al desarrollo conceptual.

Es beneficioso desarrollar la articulación de los modelos de enseñanza y aprendizaje constructivistas con las exigencias de la evaluación del sistema escolar. Ello tiene implicancias en los modelos de formación continua e inicial de los profesores, incluyendo los modelos de gestión de aula de los académicos a cargo de los cursos.

\section{Referencias}

BROUSSEAU, G. Théorie des Situations Didactiques. Grenoble: La Pensée Sauvage, 1998.

BROUSSEAU, G. Fondements et méthodes de la didactique des mathématiques. Recherches en Didactique des Mathématiques, Grenoble, v. 7, n. 2, p. 33-115, 1986.

MINEDUC (2000). Educación Matemática. Programa de Estudio Séptimo Año Básico / Nivel Básico 5. Ministerio de Educación, República de Chile, 2000. 
OLFOS, R.; GUZMÁN, I.; GALBIATI, J. Conocimiento Pedagógico del Contenido y su incidencia en la Enseñanza de la Matemática Nivel de Educación Básica. 2010. Informe Final Proyecto FONIDE N: F410980, MINEDUC, Chile. Disponible en

$<\mathrm{http} / /$ www.google.cl/url? sa $=\mathrm{t} \& \mathrm{rct}=\mathrm{j} \& \mathrm{q}=\&$ esrc $=\mathrm{s} \& \mathrm{frm}=1 \&$ source=web\& $\mathrm{cd}=1 \& \mathrm{ved}=0 \mathrm{CCsQFjAA \&}$ url $=$ http $\% 3 \mathrm{~A} \% 2 \mathrm{~F} \% 2 \mathrm{Fsgdce}$. mineduc.cl\%2Fdescargar.php\%3Fid_doc\%3D201208211303250\&ei=g6g wU47ZMeae2gWwgYGoBA\&usg=AFQjCNEVwLde2XCtUwigxZ-

pPcBbXGZQow\&sig2=NCVFI3KjdOyzAbXJ-8u1aQ>. Accesado el: 24 marzo. 2014.

RADOVIC, D.; PREISS, D. D. Patrones de Discurso Observados en el Aula de Matemática de Segundo Ciclo Básico en Chile [Discourse Patterns Observed in Middle-School Level Mathematics Classes in Chile]. Psykhe, Santiago, v. 19, n. 2, p. 65-79, 2010.

Submetido em Novembro de 2012. Aprovado em Abril de 2013. 


\begin{abstract}
ANEXO
«Le contrat didactique est la règle du jeu et la stratégie de la situation didactique; c'est le moyen qu'a le maître de la mettre en scène. Mais l'évolution de la Situation modifie le contrat qui permet alors l'obtention de situations nouvelles. De la même façon, la connaissance est ce qui s'exprime par les règles de la situation adidactique et par les stratégies. L'évolution de ces stratégies requiert des productions de connaissances qui permettent à leu tour la conception de nouvelles situations adidactiques. Le contrat didactique n'est pas un contrat pédagogique général. Il dépend étroitement des connaissances en jeu».

"... le maître cherche à faire dévolution à l'élève d'une situation adidactique qui provoque chez lui l'interaction la plus indépendante et la plus féconde possible. Pour cela, il communique ou s'abstient de communiquer, selon le cas, des informations, des questions, des méthodes d'apprentissages, des heuristiques, etc. L'enseignant est donc impliqué dans un jeu avec le système des interactions de l'élève avec le problème qu'il lui pose...»
\end{abstract}

«Topaze fait une dictée à un mauvais élève, Ne pouvant acceptée trop d'erreurs trop grossières et ne pouvant pas non plus donner directement l'orthographe demandée il suggère la réponse en la dissimulant sous des codages didactiques de plus en plus transparente...»

«La réponse que doit donner l'élève est déterminée à l'avance, le maître choisit les questions auxquelles cette réponse peut être donnée. Evidemment les connaissances nécessaires pour produire ces réponses changent leur signification aussi. En prenant des questions de plus en plus faciles, il essaie d'obtenir la signification maximum pour le maximum d'élèves. Si les connaissances visées disparaissent complètement: C'est l'effet Topaze».

"L'enseignant devait constater ce que les élèves devaient faire (et refaire) ou non, avaient appris ou avaient à apprendre. Cette activité est incontournable: on ne peut pas réduire l'enseignement à l'organisation d'apprentissages. La prise en compte " officielle " par l'élève de l'objet de la connaissance à et par le maître, de l'apprentissage de l'élève est un phénomène social important et une phase essentielle de processus didactique : cette double reconnaissance est l'objet de l'institutionnalisation. Le rôle du maître c'est aussi d'institutionnaliser!». 